\title{
Artificial Intelligence Enabled Reagent-free Imaging Hematology Analyzer
}

Xin Shu ${ }^{1}$, Sameera Sansare ${ }^{2,3}$, Di Jin ${ }^{4}$, Xiangxiang Zhang ${ }^{5}$, Kai-Yu Tong ${ }^{1}$, Rishikesh Pandey $^{2,6}$, and Renjie Zhou ${ }^{1,7}$

${ }^{1}$ Department of Biomedical Engineering, The Chinese University of Hong Kong, Shatin, New Territories, Hong Kong, China

${ }^{2}$ Connecticut Children's Innovation Center, University of Connecticut School of Medicine, Farmington, Connecticut, 06032, USA

${ }^{3}$ Department of Pharmaceutical Sciences, University of Connecticut Storrs, Connecticut, 06269, USA

${ }^{4}$ Computer Science and Artificial Intelligence Laboratory, Massachusetts Institute of Technology Cambridge, Massachusetts, 02139, USA

${ }^{5}$ School of Information Science and Engineering, Hunan University, Changsha, 410076, China

${ }^{6}$ Department of Biomedical Engineering, University of Connecticut, Storrs, Connecticut, 06269, USA

${ }^{7}$ Shun Hing Institute of Advanced Engineering, The Chinese University of Hong Kong, Shatin, New Territories, Hong Kong, China

November 1, 2021

\begin{abstract}
Leukocyte differential test is a widely performed clinical procedure for screening infectious diseases. Existing hematology analyzers require labor-intensive work and a panel of expensive reagents. Here we report an artificial-intelligence enabled reagent-free imaging hematology analyzer (AIRFIHA) modality that can accurately classify subpopulations of leukocytes with minimal sample preparation. AIRFIHA is realized through training a two-step residual neural network using label-free images of isolated leukocytes acquired from a custom-built quantitative phase microscope. By leveraging the rich information contained in quantitative phase images, we not only achieved high accuracy in differentiating B and T lymphocytes, but also classified CD4 and CD8 cells, therefore outperforming the classification accuracy of most current hematology analyzers. We validated the performance of AIRFIHA in a randomly selected test set and cross-validated it across all blood donors. Owing to its easy operation, low cost, and accurate discerning capability of complex leukocyte subpopulations, we envision AIRFIHA is clinically translatable and can also be deployed in resource-limited settings, e.g., during pandemic situations for the rapid screening of infectious diseases.

Corresponding author(s) Email: rjzhou@cuhk. edu. hk, rishikesh.pandey@uconn. edu
\end{abstract}

\section{ToC Figure}




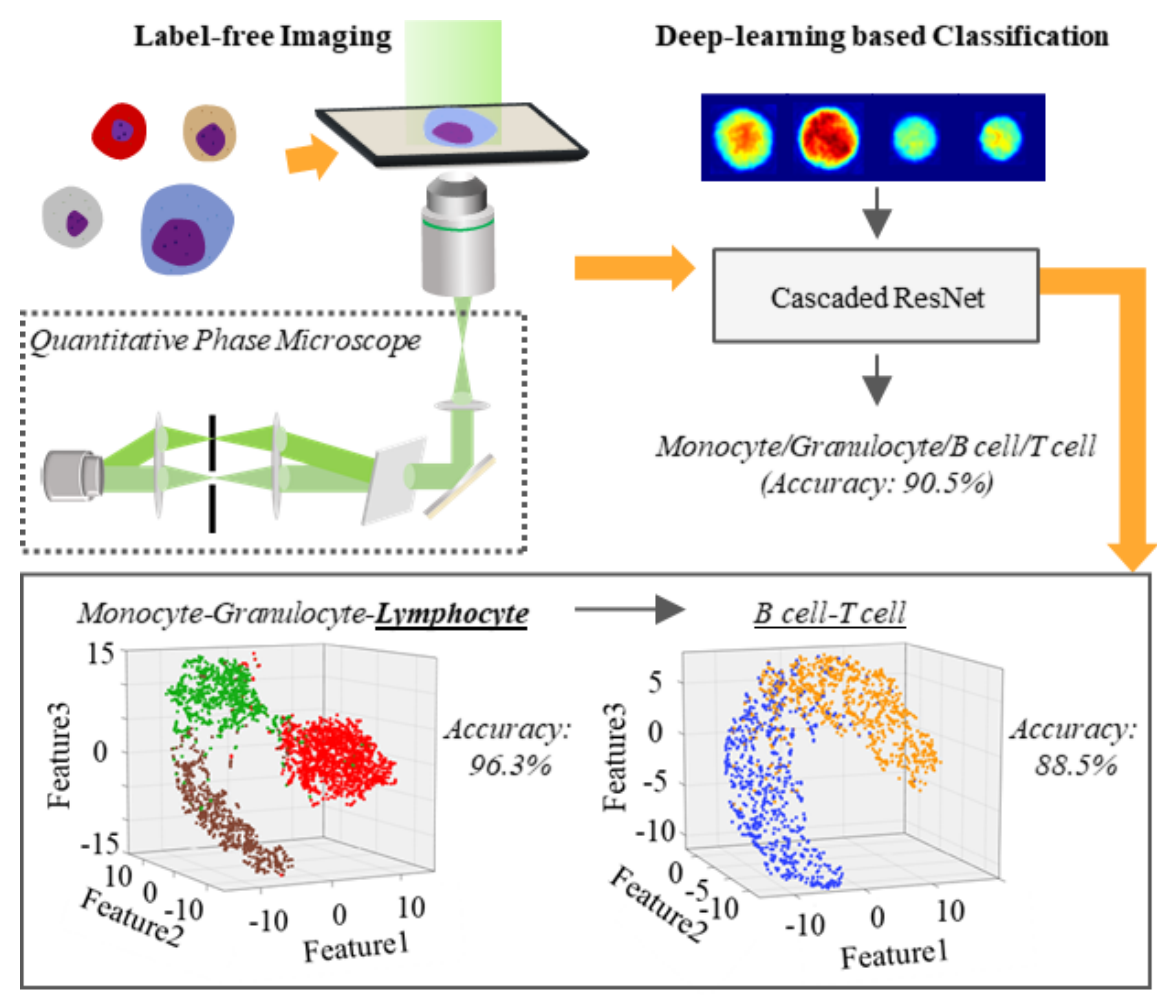

Figure 1: ToC Figure. A reagent-free hematology analyzer is developed for classifying human leukocyte subpopulations. The system is realized through a cascaded residual neural network and a quantitative phase microscope. Human B and T cells are accurately classified without using chemical labels, outperforming bright-field/dark-field based imaging methods with machine learning. This low-cost and ease-of-use method is suitable for deployment in resource-limited settings.

\section{Introduction}

Leukocytes play an important role in maintaining the normal function of human immune systems and differ in structure and function(DOnofrio and Zini, 2015; Bain, 2017). For instance, B and T lymphocytes can produce antibodies to defend the body against foreign substances, such as bacteria and viruses. Abnormal leukocyte differential counts are indications of malfunctions of the immune system or infectious diseases(Thachil and Bates, 2017). For instance, a sharp increase in neutrophil-to-lymphocyte ratio serves as an independent risk factor for SARS-CoV-2 infection(Ciccullo et al., 2020; Liu et al., 2020). To differentiate basic leukocyte types, volume and granularity parameters are often measured through electrical impedance and light scatteringbased cytometry techniques(Meintker et al., 2013; Briggs and Bain, 2017). For more complex leukocyte types with similar morphologies (e.g., B and T lymphocytes), fluorescent molecules bound with antibodies that specifically target the proteins expressed on the surface are typically used to activate fluorescence emission which can be captured by detectors for population counting. Although antibody labeling based flow cytometry methods are widely used in the clinical laboratories, there remain a few drawbacks. Firstly, only the labeled cellular structures are used for differentiating cell types, but not all the cellular structures. Secondly, an extensive list of expensive reagents is required for differentiating many cell types. Lastly, the labeled cells are not suitable for further testing as their original states have changed.

Label-free imaging methods can potentially solve the aforementioned issues(Suzuki et al., 2019; Blasi et al., 2016; Ojaghi et al., 2020; Kobayashi et al., 2017; Heo et al., 2017). For instance, a hemogram based on Raman imaging has been proposed to discern leukocytes(Ramoji et al., 2012). While this innovative approach 
leverages the unique biochemical attributes for the classification, it is limited by the weak spontaneous Raman signal, thus not suitable for high-throughput applications in a clinical setting. Quantitative phase microscopy $(\mathrm{QPM})$ is a rapidly emerging imaging modality that is suitable for high-speed imaging of unlabeled specimens. In QPM, the exact optical path-length delay associated with the density and thickness at each point in the specimen is mapped, which has enabled label-free imaging of transparent structures (e.g., live cells) with a high imaging contrast(Mir et al., 2012; Park et al., 2018; Popescu, 2011). In recent years, QPM has been used for single-cell analysis by extracting quantitative biomarkers, e.g., measuring cell dry mass to quantify cell growth(Popescu et al., 2008; Mir et al., 2011), studying red blood cell rheology(Park et al., 2010; Popescu et al., 2005), characterizing cell viability(Li et al., 2019), analyzing large cell population(Lei et al., 2018; Chen et al., 2016), and screening cancer(Zhang et al., 2020), etc. However, most studies have primarily relied on interpreting the QPM results in terms of a few principal morphological characteristics. Recently, several laboratories have sought to shift the paradigm by utilizing machine learning (ML) and artificial intelligence (AI) for analyzing and interpreting QPM data(Jo et al., 2017, 2018; Strbkova et al., 2019). As for the applications on hematology, QPM with ML/AL has been used to screen cancer cells in blood(Lee et al., 2019b; Nissim et al., 2020; Ozaki et al., 2019), diagnose red blood cell related disorders(O'Connor et al., 2020; Kim et al., 2019), detect activation states of leukocytes(Karandikar et al., 2019), and classify various leukocytes(Fanous et al., 2021; Yoon et al., 2017; Li et al., 2018; Ugele et al., 2018; Ryu et al., 2020). The full field and fast imaging attributes of QPM enable availability of volumes of high-dimension imaging and therefore make QPM a unique modality for the application of ML/AI to those tasks involving cell classification and imaging.

With recent developments in ML/AI, e.g., visual geometry group (VGG)(Simonyan and Zisserman, 2015), inception(Szegedy et al., 2015), and residual neural network (ResNet)(He et al., 2016a,b), abundant training data is available to train a model to extract important image features to classify targeted objects(Bishop, 2006; Goldberg and Holland, 1988). Compared with previous manual feature extraction analysis methods, the new approaches in ML/AI may offer features with statistically significant higher sensitivity and specificity. Among the recent ML/AI methods, ResNet tackles the gradient vanishing problem by creating shortcut paths to jump over layers. Conversion among different types of biomedical images and the segmentation of certain cell structures have been achieved by using ResNet building blocks(Rivenson et al., 2019; Lee et al., 2019a; Wang et al., 2019). With such exciting developments, ML/AI have also been applied to label-free imaging systems to tackle complicated cell analysis problems. For instance, machine learning for the differentiation of lymphocytes has been achieved on a bright-field and dark-field microscopy platform(Nassar et al., 2019) and a QPM platform using fixed pathology slides(Fanous et al., 2021). To further improve the detection accuracy and specificity of leukocyte subtypes, the method using 3D QPM technique has been proposed and demonstrated(Jo et al., 2017; Habaza et al., 2017).

In this work, we propose a rapid, low-cost, AI-enabled reagent-free imaging hematology analyzer (AIRFIHA) that can classify hierarchical leukocyte subtypes in human blood samples. Note that preliminary results of this study were presented at a conference in April 2019(Shu et al., 2019). AIRFIHA is based on leveraging the morphological attributes of phase images from a custom-built QPM system and a cascaded-ResNet for leukocyte classification. From this proof-of-principle study on six human donors, we have achieved a classification accuracy of $90.5 \%$ on average for monocytes, granulocytes, and B and T lymphocytes. The robustness and applicability of our proposed method have been confirmed by conducting cross-donor validation experiments. We further investigated the potential of AIRFIHA in discerning human CD4 and CD8 T cells. AIRFIHA demonstrated a much higher accuracy when compared with methods based on negative isolated leukocyte classification and a comparable or better accuracy when compared with methods based on positive isolated leukocyte classification. This study shows a promising perspective when applying AIRFIHA for automated clinical blood testing applications, which is especially useful in resource-limited settings and during pandemic situations. 


\section{Results}

\section{AIRFIHA system}

In this work, the classification of human leukocyte types is achieved using a QPM system and a neural network, as conceptually illustrated in Figure 1. The exact configuration of the QPM system is based on a diffraction phase microscope (DPM)(Popescu et al., 2006; Bhaduri et al., 2014; Pandey et al., 2019), which can provide highly stable and accurate phase imaging of cells. The imaging resolution of the QPM system is $590 \mathrm{~nm}$, while the field of view is around $61 \mu \mathrm{m} \times 49 \mu \mathrm{m}$. Compared with optical diffraction tomography(Yoon et al., 2017; Habaza et al., 2017), QPM does not necessitate a complex imaging system and expensive computation requiring a large amount of data, and the system is relatively cost-effective with a smaller footprint. To obtain the dataset for neural network training and classification, the leukocyte samples were isolated from the fresh blood samples of six healthy donors within 24 hours of blood extraction. The blood sample used for the leukocyte separation for each donor was in 1-3 ml range, depending on the minimum volume requirement as per manufacturer's instruction for the leukocyte subpopulations. To minimize the influence of labeling on cell activity, the leukocytes were negatively isolated by using antibodylabeled magnetic particles as illustrated in Figure 1 (a) (refer to detailed sorting procedure in "Methods"). Then, the isolated sample was diluted in PBS (phosphate buffer saline) and mounted between two glass coverslips before placing it onto a home-built QPM system as illustrated in Figure 1(b) (refer to the detailed sample preparation procedure in "Methods"). Phase images of each leukocyte type were retrieved from the measured interferograms (refer to the detailed description of the QPM system and the phase retrieval method in Supplementary). After thousands of phase images of labeled leukocytes of different types were measured, all the leukocytes in each phase image were segmented to construct the training and testing dataset(Jin et al., 2017). A neural network was constructed, trained, and validated for classifying the leukocytes using the phase image dataset (Figure 1c). A detailed description of the neural network is provided in the following section. Finally, the AIRFIHA system was used to identify leukocyte types of new samples (Figure 1d).

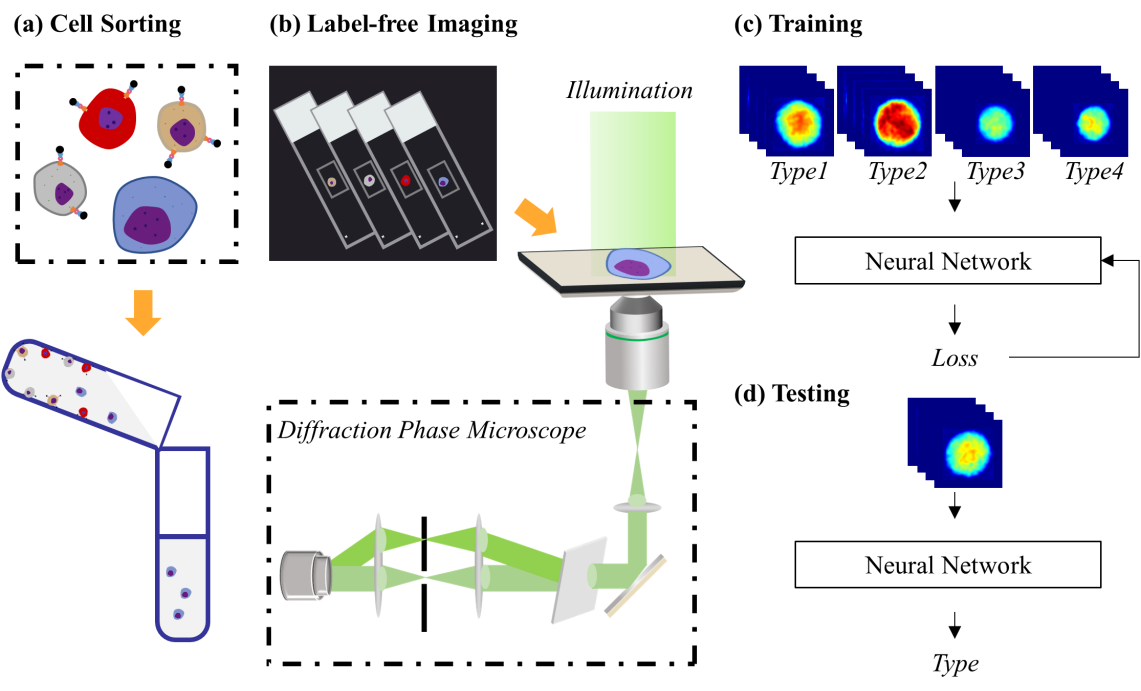

Figure 2: Workflow of the AIRFIHA system. a, Different types of leukocytes are negatively separated using antibody-labeled magnetic particles. b, A diffraction phase microscope is used for obtaining quantitative phase images of sorted leukocytes. c, Deep learning model is trained for classifying the leukocyte types. d, A trained neural-network model is used to predict leukocyte types. 


\section{Leukocyte classification method}

Phase maps of labeled leukocytes of four different types from multiple donors were measured to construct the main dataset, including 857 monocytes, 738 granulocytes, 700 B lymphocytes, 821 T lymphocytes, 211 CD4 cells, and 220 CD8 cells. Representative phase maps for each leukocyte subtype are shown in Figure 2a. Based on these phase maps, area and dry mass distributions were generated for all the leukocyte types (Figure $2 \mathrm{~b}, \mathrm{c})$. Note that cell dry mass quantifying the total protein content in a cell can be precisely determined from the phase map, and it has been well explored for cell phenotyping(Popescu et al., 2008; Barer, 1952). As shown in Figure 2b, c, monocytes and granulocytes have similar areas but very different dry masses (p-value $<0.001$ ), while they are well separated from all the other lymphocytes (B and T lymphocytes and CD4 and CD8 cells) through both area and dry mass distributions (p-value $<0.001$ ). For the main subtypes of lymphocytes, i.e., B and T lymphocytes, they are different in both cell area and dry mass (p-value $<0.001)$, but the differences are small. The subtypes of T lymphocytes, i.e., CD4 and CD8 cells, have similar cell dry mass and slightly different cell area distributions ( $\mathrm{p}$-value $<0.001$ ).

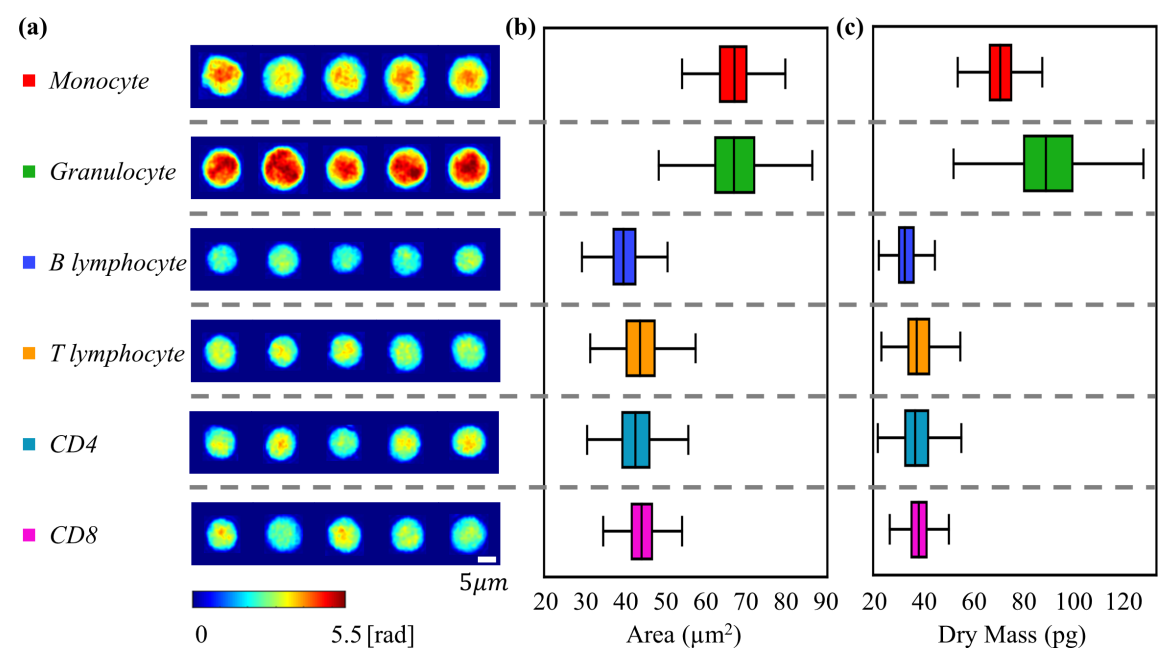

Figure 3: Representative phase images, area, and dry mass distributions of different leukocyte types. a, Representative phase images for each leukocyte type. b, c, Area and dry mass distributions for all the leukocyte types in the dataset. Monocytes (red), granulocytes (green), B lymphocytes (blue), T lymphocytes (orange), CD4 cells (light blue) and CD8 cells (purple). Scalebar: $5 \mu \mathrm{m}$.

To achieve a higher accuracy in classifying leukocytes (i.e., monocytes, granulocytes, and B and T lymphocytes), we developed a cascaded ResNet structure with a two-step classification design as shown in Figure 3a, b. This design has been inspired by the prior knowledge of leukocyte hierarchy classification structures(DOnofrio and Zini, 2015; Bain, 2017). The first ResNet (Figure 3a) is used to classify monocytes, granulocytes, and lymphocytes. The predicted lymphocytes are then put into the second ResNet (Figure $3 \mathrm{~b}$ ) for further classification into B and T lymphocytes. From this cascaded structure, we can achieve a much higher accuracy in differentiating B and T lymphocytes, compared with a single-step classifier (refer to Table S1 and S2 for more comprehensive details on the comparison of two individual donors). ResNets of different depths were explored, while the highest validation accuracy was obtained on the ResNet-10 that had around 1.5 million trainable parameters. ResNet-10 has 10 layers, i.e., one input convolution layer, 8 convolution layers from 4 building blocks (each building block has 2 convolution layers), and one final dense layer. The shortcut connects the head and tail of each building block, which helps to restore the crucial shallower features for prediction. The layer size is halved, and the kernel quantity is doubled for every 1, 2, 1 building blocks. Batch normalization (Batch Norm)(Ioffe and Szegedy, 2015) is applied for each mini-batch after each convolutional layer. Rectified Linear Unit (Relu)(Nair and Hinton, 2010) is used as the nonlinear 
activation function. After the last building block, an average pool and a flatten layer are applied to convert each two-dimensional feature map into one value, thus for 256 feature maps, a $256 \times 1$ vector is obtained to represent each of the input images. Probabilities of each type are produced based on this feature vector via a dense layer with the Softmax activation function(Bridle, 1990). For the monocyte-granulocyte-lymphocyte classification task, probabilities of these three types are produced, while for B-T lymphocyte classification, two probability values are produced. The type with the largest probability value is used to make the final decision. To explore the differentiation capability of CD4 and CD8 cells, a separate ResNet was trained by fine-tuning the B-T lymphocyte classifier for the new classification task (Figure 3c). Details on the training and validation of the classification model are provided in the Method section.

\section{Classification results}

To test the classification capability of our AIRFIHA system, a test set was first constructed by randomly selecting 100 cells from four leukocytes, i.e., monocytes, granulocytes, and B and T lymphocytes. Notably, the test set was not contained in the training set. The classification results were evaluated using recall, precision, and F1-score(Powers, 2011). F1-score, which is the harmonic mean of recall and precision, is used to characterize the final classification result. The F1-scores from the first classifier for monocytes, granulocytes, and lymphocytes are $94.0 \%, 95.4 \%$, and $97.7 \%$, respectively (detailed numerical values for recall, precision, and F1-scores are provided in Table S3). The F1-scores from the second classifier for B and $\mathrm{T}$ lymphocytes are $88.2 \%$ and $88.8 \%$, respectively (detailed numerical values for recall, precision, and F1-scores are provided in Table S4). The overall detection results are summarized and visualized in Figure 4a and Table S5. The precision-recall curves(Saito and Rehmsmeier, 2015) for each of the classifiers in the cascaded-ResNet are plotted and shown in Figure 4b and 4c. The values of the area under the precisionrecall curve (AUPRC) for lymphocytes, monocytes, and granulocytes in the first classifier are 1.00, 0.98 and 0.98, respectively. The values of AUPRC for B and T lymphocytes in the second classifier are 0.96 and 0.94 , respectively. Our B/T cell classification accuracy is comparable with the method based on 3D quantitative phase imaging (note that leukocytes here were from one mice that could make a difference on the accuracy)(Yoon et al., 2017).

The prediction from the ResNets is based on the feature vectors which are placed at the end of convolutional layers. The model should produce similar feature vectors for the same input types and very different feature vectors for different input types. To verify the efficacy of our trained ResNets, the t-distributed stochastic neighbor embedding (t-SNE) method(Maaten and Hinton, 2008) was used, which has decreased the feature dimension from 256 to 3 for all the cell types. The features are plotted in the same coordinate space as shown in Figure 4d, e. ResNet extracted features could help to distinguish B and T lymphocytes. Compared with principal component analysis (PCA) method, those features have made the boundaries between $\mathrm{B}$ and $\mathrm{T}$ lymphocytes clearer (refer to Figure S3 in Supplementary Material). As summarized in Table S7 in Supplementary Material, the ResNet method shows increased classification accuracy by over $15 \%$ for B and T lymphocytes compared with the PCA method, as evaluated with a trained support vector machine (SVM) classifier. To understand what differences in the cell morphological features contributed to the discrimination in the neural network, we visualized the outputs of each convolutional layer after activation (refer to Figure S4 in Supplementary Material). From the last layers of analyzed leukocytes, we observed that the output features are mainly focused on the nucleus, cytoplasm, and membrane characteristics, elucidating that these cellular features are important in classification. These observations are consistent with the knowledge that subtypes of leukocytes differ in their nuclear, cytoplasmic, and membrane attributes(DOnofrio and Zini, 2015; Bain, 2017). To explore the cause of classification errors, some mistakenly classified cells are listed out. Apart from the morphological similarities between different cell types, the error could be also caused by the mislabeling in the ground truth dataset (refer to Figure S5 in Supplementary Material). To determine the optimum numbers of cells for achieving a stable and high detection accuracy, we have analyzed the detection accuracy vs. total number of cells used for training (refer to Figure S6 in Supplementary Material). It is 


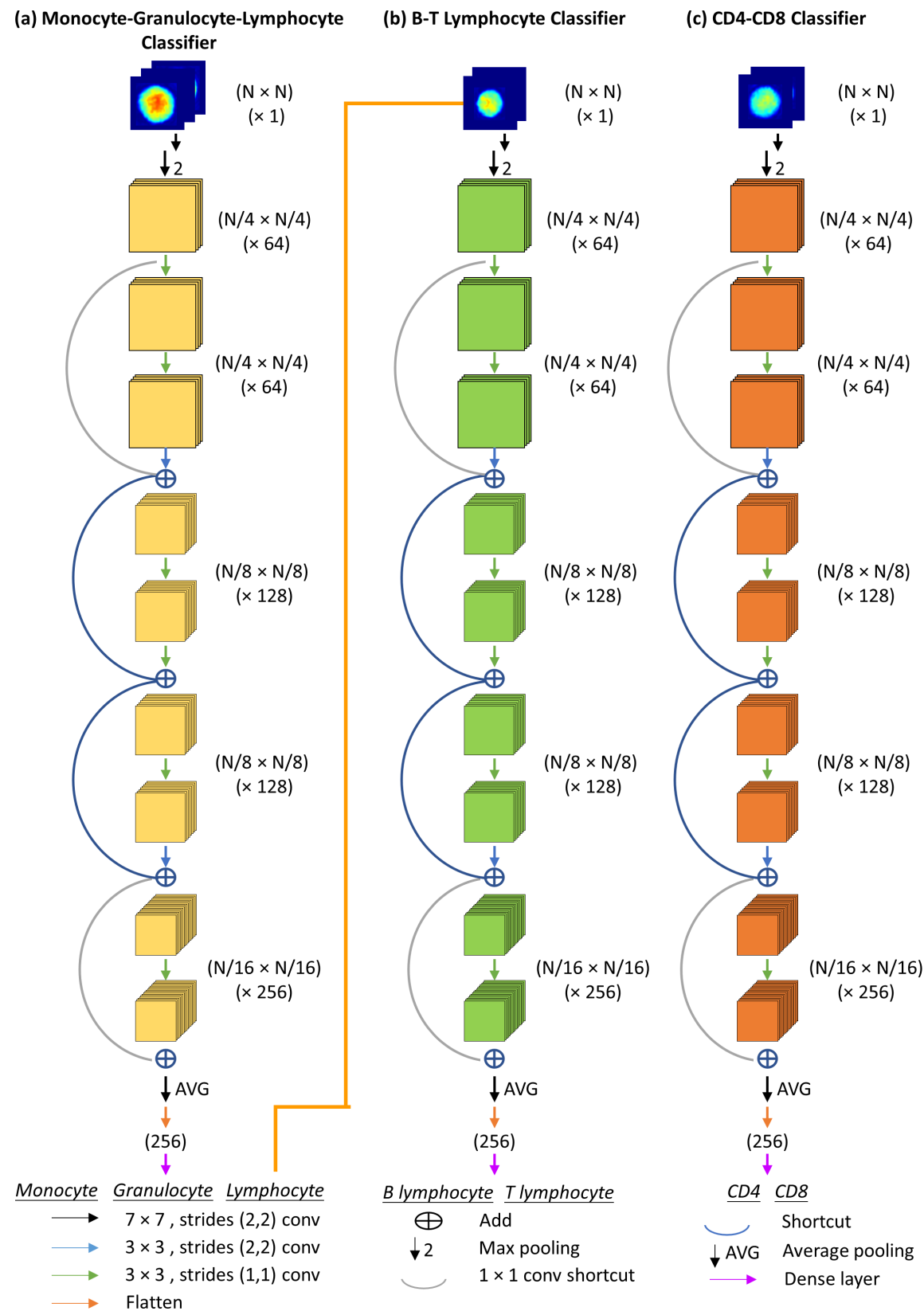

Figure 4: The ResNets constructed for classification of leukocytes. a, b, The cascaded-ResNet-10 for classifying monocytes, granulocytes, and B and T lymphocytes. c, The ResNet-10 for classifying CD4 and CD8 cells.

found that the detection accuracy becomes stable when the training dataset is $>800$ cells (i.e., $>200$ cells per type). In this study, we have collected a total of 2,700 cells with $>600$ cells in each type for training, which was appropriate for achieving a reliable classification result.

CD4 and CD8 cells are subtypes of T lymphocytes and have very similar morphological features(Yoon et al., 2017). Routine monitoring of CD4/CD8 cell ratio with point-of-care systems helps monitor immunodeficiency related diseases, e.g. acquired immunodeficiency syndrome (AIDS)(Jani et al., 2011; Boyle et al., 
(a)

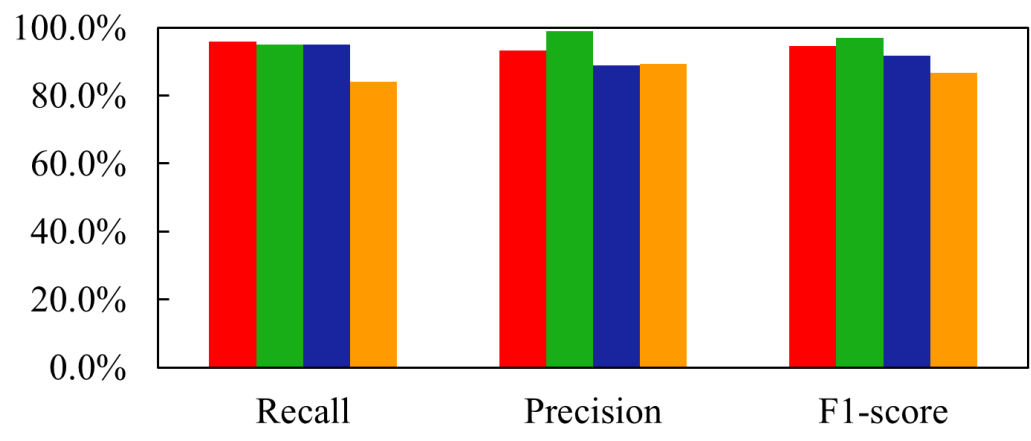

(b)

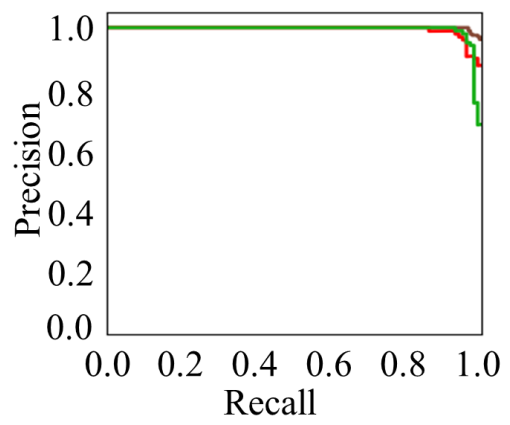

(d)

(c)

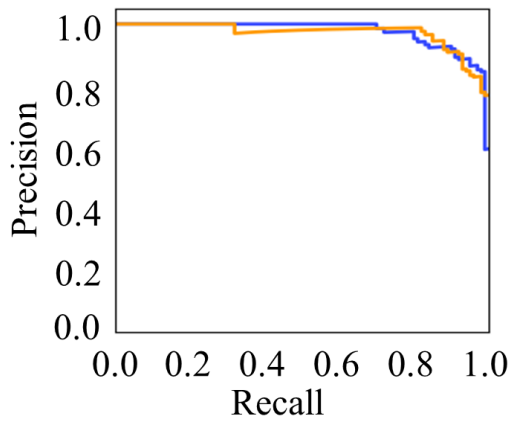

(e)

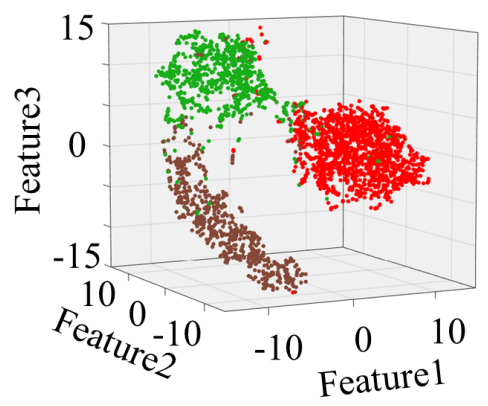

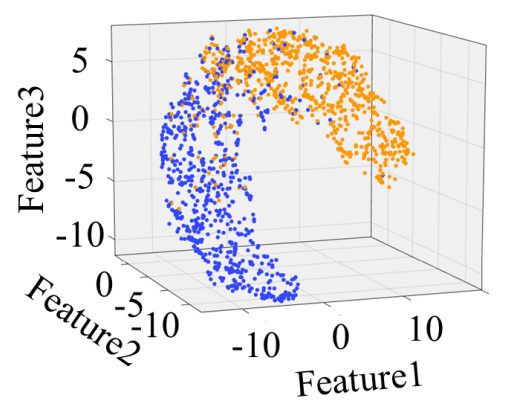

(f)

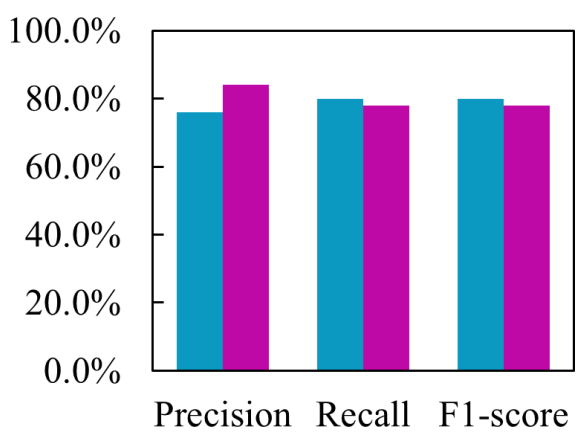

(g)

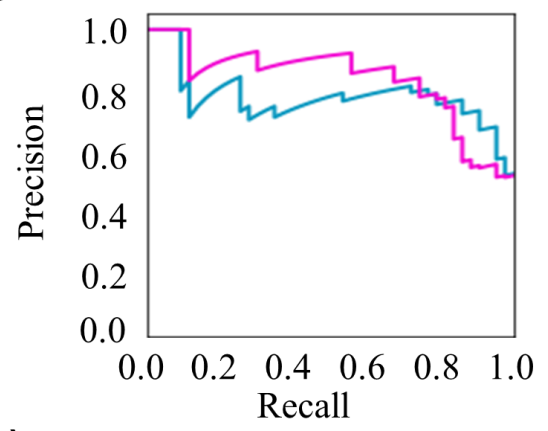

(h)

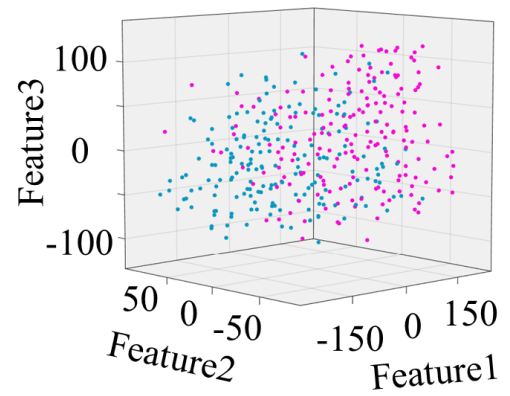

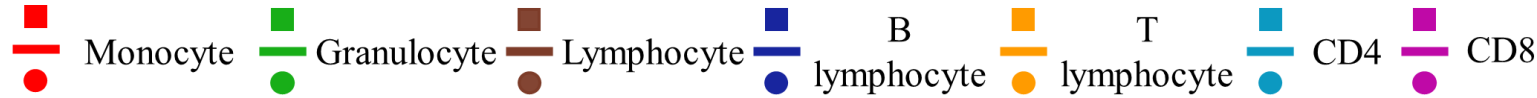

Figure 5: Figure 4. Classification results on monocytes (red), granulocytes (green), B lymphocytes (blue), T lymphocytes (orange), CD4 cells (light blue), and CD8 cells (purple). a, Recall, precision, and F1-score for four types of leukocytes. b, Precision-recall curves for the monocyte-granulocyte-lymphocyte classifier. c, Precision-recall curves for the B-T lymphocyte classifier. d, e, T-SNE visualization of the feature extracted by the above two classifiers. f, Recall, precision, and F1-score for CD4 and CD8 cells. g, Precision-recall curve for the CD4-CD8 classifier. h, T-SNE visualization of the feature extracted by the CD4-CD8 classifier.

2012). Our proposed AI-powered platform has the potential to offer a unique approach in which the T cells can be virtually isolated and subtyped while also preserving them for subsequent immunophenotypic analysis. Moreover, such a platform can be expanded to visualize the immunological responses due to its label-free attributes. We had previously demonstrated the use of QPM in identifying the activation state of CD8 cells 
in a contrast-free manner(Karandikar et al., 2019). Building up on our previous study, we conjectured that our QPM can be used for differentiating CD4 and CD8 cells in a label-free manner. To test our hypothesis, we employed our AIRFIHA system on CD4 and CD8 cells from the same blood donor for both training and testing. The classification result is summarized in Figure 4f-h. F1-scores of $80.4 \%$ and $77.5 \%$ for CD4 and CD8 cells are achieved, respectively (detailed values for recall, precision, and F1-scores are provided in Table S6). Compared with the F1-scores of $85.7 \%$ and $88.8 \%$ for CD4 and CD8 cells obtained by using 3D refractive maps(Yoon et al., 2017), our preliminary results have a bit lower accuracy. The AUPRC values for CD4 and CD8 cells are 0.78 and 0.84, respectively. Using the t-SNE method, features are extracted from the CD4-CD8 classifier and plotted (Figure 4h) for visualizing the differentiation capability. Our preliminary results show that our method has a basic differentiation capability for these two subtypes of $\mathrm{T}$ lymphocytes. The accuracy can be increased by using high volume of data and further tuning of our neural network.

\section{Cross-donor and intra-donor validation}

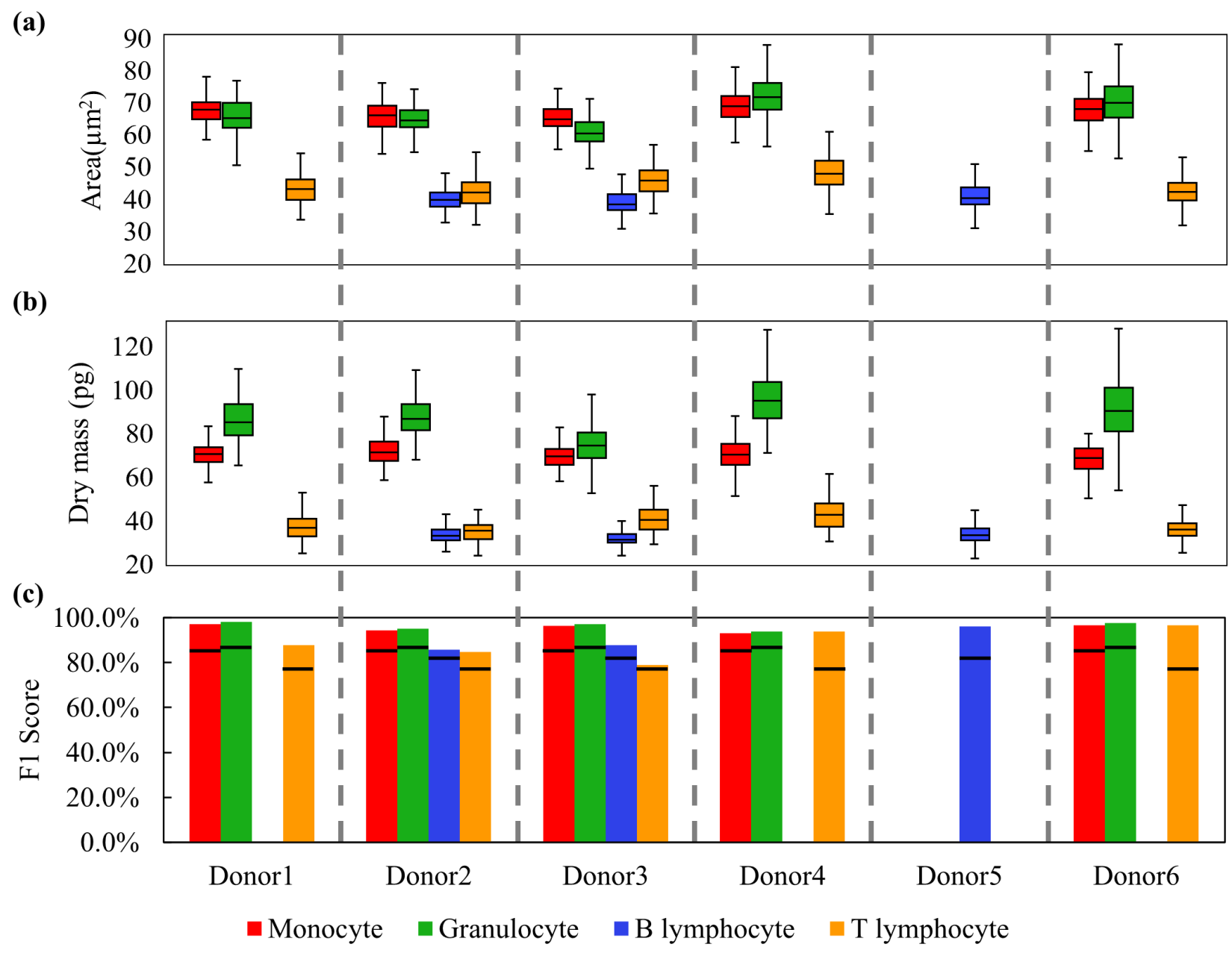

Figure 6: a, Comparison of leukocyte area distributions among different donors. Monocytes (red), Granulocytes (green), B lymphocytes (blue), T lymphocytes (orange) from 6 donors are included. b, Comparison of leukocyte dry mass distributions among different donors. c, F1-Score of leukocyte subtypes of each test donor from the cross-donor validation experiment.

As for real clinical applications, the blood test samples normally come from new individuals whose blood 
samples will not be known by our model. There could be variances in the morphological features of leukocytes of each type between different donors, depending on their age, health status, etc(Olsson et al., 2001; Brown et al., 2006). To verify whether such variances exist among our donors, we plotted the area and dry mass distributions for each donor (Figure 5a,b), from which it was found out that there were indeed distribution differences between donors for certain leukocyte types (note that for several donors the distributions for certain types of leukocytes were missing). Since we had already acquired QPM images as a part of another work involving B cell leukemia, we decided not to measure the B cells from all the donors. This partly helped in ensuring that the extraction and subsequent QPM measurement from all the other leukocytes were completed within 3-4 hours of receiving the samples. In any case, we have more than 3 different donors for every leukocyte sample and we believe that it is sufficient for this proof-of-the-concept investigation. The effect of such differences on the generalization of our model to the new donor was explored. For this purpose, the leukocyte samples from five donors were used for training ( $>200$ cells per type) and the leukocyte samples from the remaining donor were used for testing. This experiment was repeated by rotating the testing donor in the same group. The classification result is plotted in Figure 5c (detailed numerical values are provided in Table S8-S10). The result obtained earlier using all six donors (refer to Figure 4a) is set as the reference for comparison. As for monocyte and granulocyte, all variances of the 10 tests are less than 0.1. For B lymphocytes only 1 out of 3 tests has variances larger than 0.1 , while for $\mathrm{T}$ lymphocytes only 1 out of 5 tests has a variance larger than 0.1. The over-all average F1-scores for monocytes, granulocytes, and $\mathrm{B}$ and $\mathrm{T}$ lymphocytes are $91.6 \%, 94.3 \%, 83.7 \%$, and $81.4 \%$, respectively. Notable, the average F1-scores for the B-T lymphocyte classifier of the cascaded-ResNet are $84.1 \%$ and $85.9 \%$. This has demonstrated a better accuracy when compared with an earlier work on the classification of $\mathrm{B}$ and $\mathrm{T}$ lymphocytes, where average F1-scores of $79.4 \%$ and $75.7 \%$ were obtained, respectively, using bright-field and dark-field imaging cytometry systems(Nassar et al., 2019). To further understand the feasibility of our method, we performed an intra-donor analysis to reveal the variations among leukocytes within the same donor (refer to the F1-scores summarized in Table S11 in Supplementary Material). The results show that there are variations within the same donor, albeit small on average. The cross-donor and intra-donor validation results have shown that our method has a high potential for clinical applications.

\section{Discussion}

In this proof-of-concept study, the capability of AIRFIHA for label-free classification of leukocyte subpopulations has been demonstrated on human blood donors. With a well-designed neural network model, high information-content quantitative phase images, and a considerable amount of data collected from human blood donors, our AIRFIHA method has outperformed current reagent-free methods for the classification of granulocytes, monocytes, and B and T lymphocytes. Our preliminary result also shows that the detection accuracy of our method is not severely affected by different donors, thus indicating a potential for use in clinical settings. We have further demonstrated that AIRFIHA can differentiate CD4 and CD8 cells that are normally difficult to distinguish with label-free methods.

Error Analysis. It is important to note that our classification results rely on the accuracy of the separation kits used in this study to select the individual sets of leukocytes. We employed flow cytometry (refer to the details in "Methods") to measure the percentage population of the specific leukocytes after isolating them using the corresponding kits and the representative results from a donor are presented in Supplementary Figure S7. These negative isolation kits have inherent inaccuracy that can adversely affect the classification results. However, compared with positive selection kits, negative selection kits could better maintain the original cell morphology for our label-free imaging modality, where the morphological attributes form the basis for classification.

Result Evaluation. We compared our result with other reported results using different detection/imaging principles, labeling methods, and experiment instruments, as shown in Table S12 in Supplementary Ma- 
terial. AIRFIHA has a significantly improved accuracy when compared with the methods based on negative isolated leukocyte classification(Ugele et al., 2018). For the classification of monocytes, granulocytes, and lymphocytes, our detection accuracy is slightly lower than the methods using positive fluorescence sorting or complicated purification methods(Li et al., 2018; Nassar et al., 2019; Wu et al., 2016). It is possible that the negative selection kits have intrinsic lower accuracies in isolating leukocytes when compared with using positive kits, therefore reducing our classification accuracy. If there is a way to sort the leukocytes with higher accuracies without affecting the original morphology states of cells, we expect to further increase the classification accuracy. For the classification of B and T lymphocytes, our result is better than bright and dark field microscopy-based methods for the cross-donor validation experiments(Nassar et al., 2019). To a certain extent, our method benefits from the subtle differences in the refractive index maps of intracellular structure as encoded in the quantitative phase maps. Our classification accuracy is also comparable with 3D QPM based methods that explore expensive and complex instrumentations (note that no human blood test and cross-donor validation have been carried in such methods so far)(Yoon et al., 2017). Notably, both mentioned methods are based on using positive leukocyte extraction methods. As for the classification of CD4 and CD8 cells, our classification accuracy is also compared with that obtained using 3D QPM methods(Yoon et al., 2017).

Further Improvement. With the capability to differentiate very complex leukocyte types, AIRFIHA can provide more comprehensive information for potential disease diagnoses with simplified testing procedures. There are still ways to improve the detection accuracy of our system, such as improving the phase imaging resolution through synthetic aperture phase imaging method(Zheng et al., 2013), deconvolution(Li et al., 2017), and using 3D-resolved phase maps, preferably captured through a single image acquisition to avoid taking a large amount of data (such method has been recently made possible; a manuscript is under preparation)(Ge et al.). The other way to improve accuracy is to expand the dataset and upgrade the neural network model.

Potential Applications. Overall, our results show the potential of AIRFIHA as a fully automated, reagentfree, and high-throughput modality for differential diagnosis of leukocytes at point-of-care and in a clinical laboratory. Additional salient features of this platform include its single-shot measurement, small spatial footprint, and low cost. Of note, owing to its facile and simpler set-up, this platform can be combined with other modalities for blood cell investigation. For example, by combining it with microfluidic devices, AIRFIHA can conduct blood testing and analysis in a fully automated way. Importantly, the need for isolation kits is obviated and the leucocytes separated from blood using a routine centrifugation process can be directly subjected to the AIRFIHA to provide percentage population of leukocyte subtypes. One other example could be its integration with Raman spectroscopy that has been proposed for B lymphocytes acute lymphoblastic leukemia identification and classification(Managò et al., 2016). While Raman spectroscopy provides biomolecular specificity, spontaneous Raman measurements are not feasible for clinical workflow requiring rapid diagnosis. Importantly, given the potential of the AIRFIHA platform in screening the B cells from other leucocytes, this QPM-based strategy can be used to screen the B lymphocytes where Raman measurements can be performed for B lymphocytes leukemia diagnosis. The combined QPM-Raman system obviates the need of any additional separation method to select B lymphocytes either from the blood or from the leucocyte mixtures for leukemia diagnosis in a label-free manner. Moreover, as AIRFIHA involves a low-cost system that requires minimal sample preparation or chemical consumables, our AIRFIHA has a great potential to be used in point-of-care applications, resource-limited settings, or pandemic situations, e.g., COVID-19 pandemic, in view of a portable and low-cost QPM system recently demonstrated by us(Niu et al., 2020).

\section{Method}

Fresh blood sample procurement. The fresh blood samples from six anonymous healthy adult donors were purchased from StemCell Technologies (Vancouver, Canada) and all the experiments were conducted 
within 24 hours of blood extraction. The purchased blood samples contained ethylenediaminetetraacetic acid (EDTA) as the anti-coagulant.

Leukocyte isolation from fresh blood. Four types of leukocytes, namely monocytes, granulocytes, and B and T lymphocytes, were isolated from fresh blood samples using isolation kits from Stemcell Technologies. From each donor the amount of blood was in the 1-3 $\mathrm{ml}$ range, depending on the minimum volume requirement as per manufacturer's instruction for each leukocyte subpopulation. To isolate these four subpopulations, we used EasySep Direct Human Monocyte Isolation Kit, EasySep Direct Human PanGranulocyte Isolation Kit, EasySep Direct Human T Cell Isolation Kit, and EasySep Direct Human B Cell Isolation Kit (Stemcell Technologies Inc). These separation kits used immunomagnetic negative selection for isolating each specific leukocyte type from the whole blood sample. Two additional negative separation kits, i.e., EasySep Direct Human CD4+ T Cell Isolation Kit and EasySep Direct Human CD8+ T Cell Isolation Kit, were used for the isolation of CD4 and CD8 cells, respectively. Phosphate-buffered saline free from $\mathrm{Ca}++$ and $\mathrm{Mg}++$ (Gibco, Thermo Fisher Scientific) was used as the recommended medium for the EasySep Isolation kits. The isolation was carried out following the manufacturer's instructions with multiple cycles of mixing and incubation with the provided RapidSpheres and cocktail from the isolation kits. The final incubation yielded the isolated leukocytes in a $14 \mathrm{ml}$ polystyrene round-bottom tube (Thermo Fischer Scientific), which were centrifuged at $400 \mathrm{~g}$ for 5 minutes. The cell pellet was resuspended in PBS before the cells were imaged.

Flow cytometry analysis. Flow cytometry was performed on the isolated leukocytes after the EasySep procedure to confirm the purity of the isolation. The viability of the leukocytes was checked with Acridine Orange and Propidium Iodide (AO/PI) staining (Invitrogen, Thermo Fischer Scientific) using a cell counter. The isolated leukocytes were counted and 50,000 of them were resuspended in cold PBS (Gibco, Thermo Fisher Scientific) at a density of $10^{7} / \mathrm{ml}$. $100 \mathrm{ml}$ of this cell suspension was added to each well in a 96 well plate. $1 \mu \mathrm{l}$ of the required fluorophore-conjugated antibody was added to each well and incubated in the refrigerator for 20 mins. Anti-CD-14- PerCP was used for monocytes, Anti-CD-66b-FITC was used for granulocytes, Anti-CD-19- APC was used for B lymphocytes, and Anti-CD3- PE was used for T lymphocytes. The leukocytes were washed thrice with cold PBS and resuspended in $100 \mu$ of cold PBS. The leukocytes were used for the flow cytometry analysis (MACSQuant Analyzer) and the data were analyzed with FlowJo software.

Leukocyte sample preparation for quantitative phase imaging. After the isolation of the leukocytes, we suspended them in PBS solution and diluted five-ten times. DNase solution $(1 \mathrm{mg} / \mathrm{ml})$ (Stemcell Technologies Inc) was added to the isolated cells to decrease the clumping and adsorption of protein fragments. Typically, $10 \mu \mathrm{l}$ of the isolated cell suspension was sandwiched between two quartz coverslips and a secure seal spacer. Then, the sample was placed onto the sample-stage of the home-built system for quantitative phase imaging. We repeated this sample preparation procedure for collecting all the required phase images of leukocytes from each donor.

Training of the classification model. Phase maps of the leukocytes were obtained by cropping the phase images retrieved from the measured interferograms. Each phase map, containing one leukocyte, was then resized to $300 \times 300$ pixels to be used as the input of the network. In the training process, a 5 -fold crossvalidation method was used to tune the hyperparameters, including network depth, batch size, learning rate, etc. During the training, to ensure all leukocyte types were trained under the same condition (i.e., each type has the same number of training samples), the datasets of unbalanced leukocyte types were augmented by rotation, position shifting, and flipping. For the monocyte-granulocyte-lymphocyte classifier, $\mathrm{B}$ and $\mathrm{T}$ lymphocytes were treated as one type, i.e., lymphocytes, and then all granulocytes, monocytes and lymphocytes were used to train and test the classifier. Categorical cross-entropy loss and Adam optimizer (learning rate $=1 * 10^{-3}, \beta_{1}=0.9, \beta_{2}=0.999$, learning rate decay $\left.=0\right)($ Kingma and Ba, 2017) were applied to optimize the model. In the end, the model with the best average validation accuracy was chosen as the final monocyte-granulocyte-lymphocyte classifier. For the B-T lymphocyte classifier, the dense layer of the obtained monocyte-granulocyte-lymphocyte classifier was first replaced with a new dense layer that has two 
outputs. All the B and T lymphocytes were used to fine-tune the entire network. Categorical cross-entropy loss and SGD optimizer (learning rate $=1 * 10^{-3}$, learning rate decay $=1 * 10^{-6}$, momentum $\left.=0.9\right)($ Robbins and Monro, 1951) were used. The network model with the best validation result was chosen as the final B-T lymphocyte classifier. By connecting these two network models, the final cascaded network model was obtained, from which the testing was conducted. The CD4-CD8 classifier was fine-tuned from the B-T lymphocyte classifier and trained and tested within the same donor. These frameworks were implemented with Tensorflow backend Keras framework and Python in the Microsoft Windows 10 operating system. The training was performed on a computer workstation, configured with an Intel i9-7900X CPU, 128 GB of RAM, and an Nvidia Titan XP GPU.

\section{Acknowledgements}

This research project was supported by Innovation and Technology Commission - Hong Kong (Award Numbers: ITS/394/17 and ITS/098/18FP), Croucher Foundation (Award Number: $\mathrm{CM} / \mathrm{CT} / \mathrm{CF} / \mathrm{CIA} / 0688 / 19 \mathrm{ay}$ ), Shun Hing Institute of Advanced Engineering (Award Number: BMEp3-18), and CUHK Research Sustainability of Major RGC Funding Schemes (RSFS) - Strategic Areas Information and Automation Technology area. The authors are grateful to Prof. Pramod Srivastava for allowing us to use the flow cytometry facility and Dr. Sukrut Karandikar for his help with flow cytometer measurements. The authors are also thankful to Rosalie Bordett and Tiffany Liang for their help with the cell sorting experiments.

\section{Author Contributions}

R.P., and R.Z., conceived the original idea and directed the whole research work. R.Z., and R.P., designed and built the quantitative phase microscope. S.S. performed leukocyte isolation and imaging experiments following guidance from R.P. X.S., designed, implemented, and optimized the classification models and analyzed the results following guidance from R.Z. D.J., and X.Z., provided guidance on the optimization of the classification models. X.S., and R.Z., wrote the manuscript with contribution from all the authors.

\section{Conflict of Interest}

A US provisional patent has been filed. R. Z. has financial interests in Bay Jay Ray Technology Limited.

\section{Data Availability Statement}

The data that support the findings of this study are available from the corresponding authors upon reasonable request.

\section{References}

Barbara J. Bain. Blood Cell Morphology in Health and Disease, book section 5, pages 61-92. Elsevier, 2017. ISBN 978-0-7020-6696-2.

R Barer. Interference microscopy and mass determination. Nature, 169(4296):366-367, 1952. ISSN 0028-0836. 
Basanta Bhaduri, Chris Edwards, Hoa Pham, Renjie Zhou, Tan H Nguyen, Lynford L Goddard, and Gabriel Popescu. Diffraction phase microscopy: principles and applications in materials and life sciences. Advances in Optics and Photonics, 6(1):57-119, 2014. ISSN 1943-8206.

Christopher M Bishop. Pattern recognition and machine learning. Springer, 2006. ISBN 1493938436.

Thomas Blasi, Holger Hennig, Huw D Summers, Fabian J Theis, Joana Cerveira, James O Patterson, Derek Davies, Andrew Filby, Anne E Carpenter, and Paul Rees. Label-free cell cycle analysis for high-throughput imaging flow cytometry. Nature Communications, 7:10256, 2016. ISSN 2041-1723.

David S Boyle, Kenneth R Hawkins, Matthew S Steele, Mitra Singhal, and Xuanhong Cheng. Emerging technologies for point-of-care CD4 T-lymphocyte counting. Trends in Biotechnology, 30(1):45-54, 2012. ISSN 0167-7799.

John S Bridle. Probabilistic interpretation of feedforward classification network outputs, with relationships to statistical pattern recognition. Neurocomputing, 68:227-236, 1990.

Carol Briggs and Barbara J. Bain. Basic Haematological Techniques, book section 3, pages 18-49. Elsevier, 2017. ISBN 978-0-7020-6696-2.

DC Brown, CV Maxwell, GF Erf, ME Davis, S Singh, and ZB Johnson. Ontogeny of T lymphocytes and intestinal morphological characteristics in neonatal pigs at different ages in the postnatal period. Journal of Animal Science, 84(3):567-578, 2006. ISSN 0021-8812.

Claire Lifan Chen, Ata Mahjoubfar, Li-Chia Tai, Ian K Blaby, Allen Huang, Kayvan Reza Niazi, and Bahram Jalali. Deep learning in label-free cell classification. Scientific Reports, 6:21471, 2016. ISSN 2045-2322.

A. Ciccullo, A. Borghetti, L. Z. Dal Verme, A. Tosoni, F. Lombardi, M. Garcovich, F. Biscetti, M. Montalto, R. Cauda, and S. Di Giambenedetto. Neutrophil-to-lymphocyte ratio and clinical outcome in COVID-19: a report from the Italian front line. International Journal of Antimicrobial Agents, 56(2):106017, 2020. ISSN 0924-8579.

G. DOnofrio and G. Zini. Blood and Bone Marrow Cells, book section 1, pages 1-87. 2015.

Michael Fanous, Gabriel Popescu, Krishnarao Tangella, and Nahil Sobh. White blood cell detection, classification and analysis using phase imaging with computational specificity (PICS). In Proc. SPIE 11653, Quantitative Phase Imaging VII, page 1165311, 2021.

B Ge, M Deng, Y He, Yijin Wang, Md Habibur Rahman, Yiping Ho, Liting Duan, Zahid Yaqoob, G Barbastathis, P T C So, and R Zhou. Single-frame cell tomography at speed in excess of 10,000 three-dimensional images per second.

David E Goldberg and John Henry Holland. Genetic algorithms and machine learning. Machine Learning, 3:95-99, 1988. ISSN 0885-6125.

Mor Habaza, Michael Kirschbaum, Christian Guernth-Marschner, Gili Dardikman, Itay Barnea, Rafi Korenstein, Claus Duschl, and Natan T Shaked. Rapid 3D refractive-index imaging of live cells in suspension without labeling using dielectrophoretic cell rotation. Advanced Science, 4(2):1600205, 2017. ISSN 21983844 .

Kaiming He, Xiangyu Zhang, Shaoqing Ren, and Jian Sun. Deep residual learning for image recognition. In Proc. 2016 IEEE Conference on Computer Vision and Pattern Recognition, pages 770-778, 2016a.

Kaiming He, Xiangyu Zhang, Shaoqing Ren, and Jian Sun. Identity mappings in deep residual networks. In Proc. 2016 European Conference on Computer Vision, page 630-645, 2016b.

Young Jin Heo, Donghyeon Lee, Junsu Kang, Keondo Lee, and Wan Kyun Chung. Real-time image processing for microscopy-based label-free imaging flow cytometry in a microfluidic chip. Scientific Reports, 7(1):1-9, 2017. ISSN 2045-2322. 
Sergey Ioffe and Christian Szegedy. Batch normalization: Accelerating deep network training by reducing internal covariate shift. arXiv:1502.03167, 2015.

Ilesh V Jani, Nádia E Sitoe, Eunice R Alfai, Patrina L Chongo, Jorge I Quevedo, Beatriz M Rocha, Jonathan D Lehe, and Trevor F Peter. Effect of point-of-care CD4 cell count tests on retention of patients and rates of antiretroviral therapy initiation in primary health clinics: an observational cohort study. The Lancet, 378(9802):1572-1579, 2011. ISSN 0140-6736.

Di Jin, Yongjin Sung, Niyom Lue, Yang-Hyo Kim, Peter TC So, and Zahid Yaqoob. Large population cell characterization using quantitative phase cytometer. Cytometry Part A, 91(5):450-459, 2017.

YoungJu Jo, Sangjin Park, JaeHwang Jung, Jonghee Yoon, Hosung Joo, Min-hyeok Kim, Suk-Jo Kang, Myung Chul Choi, Sang Yup Lee, and YongKeun Park. Holographic deep learning for rapid optical screening of anthrax spores. Science Advances, 3(8):e1700606, 2017. ISSN 2375-2548.

YoungJu Jo, Hyungjoo Cho, Sang Yun Lee, Gunho Choi, Geon Kim, Hyun-seok Min, and YongKeun Park. Quantitative phase imaging and artificial intelligence: a review. IEEE Journal of Selected Topics in Quantum Electronics, 25(1):1-14, 2018. ISSN 1077-260X.

Sukrut Hemant Karandikar, Chi Zhang, Akilan Meiyappan, Ishan Barman, Christine Finck, Pramod Kumar Srivastava, and Rishikesh Pandey. Reagent-Free and Rapid Assessment of T Cell Activation State Using Diffraction Phase Microscopy and Deep Learning. Analytical Chemistry, 91(5):3405-3411, 2019. ISSN 0003-2700.

Geon Kim, YoungJu Jo, Hyungjoo Cho, Hyun-seok Min, and YongKeun Park. Learning-based screening of hematologic disorders using quantitative phase imaging of individual red blood cells. Biosensors and Bioelectronics, 123:69-76, 2019. ISSN 0956-5663.

Diederik P Kingma and Jimmy Ba. Adam: A method for stochastic optimization. arXiv:1412.6980, 2017.

Hirofumi Kobayashi, Cheng Lei, Yi Wu, Ailin Mao, Yiyue Jiang, Baoshan Guo, Yasuyuki Ozeki, and Keisuke Goda. Label-free detection of cellular drug responses by high-throughput bright-field imaging and machine learning. Scientific Reports, 7(1):1-9, 2017. ISSN 2045-2322.

Jimin Lee, Hyejin Kim, Hyungjoo Cho, YoungJu Jo, Yujin Song, Daewoong Ahn, Kangwon Lee, YongKeun Park, and Sung-Joon Ye. Deep-learning-based label-free segmentation of cell nuclei in time-lapse refractive index tomograms. IEEE Access, 7:83449-83460, 2019a. ISSN 2169-3536.

Kelvin C.M. Lee, Maolin Wang, Kathryn S.E. Cheah, Godfrey C.F. Chan, Hayden K.H. So, Kenneth K.Y. Wong, and Kevin K. Tsia. Quantitative Phase Imaging Flow Cytometry for Ultra-Large-Scale Single-Cell Biophysical Phenotyping. Cytometry Part A, 95(5):510-520, 2019b. ISSN 1552-4922.

Cheng Lei, Hirofumi Kobayashi, Yi Wu, Ming Li, Akihiro Isozaki, Atsushi Yasumoto, Hideharu Mikami, Takuro Ito, Nao Nitta, and Takeaki Sugimura. High-throughput imaging flow cytometry by optofluidic time-stretch microscopy. Nature Protocols, 13(7):1603-1631, 2018. ISSN 1750-2799.

Jizhou Li, Florian Luisier, and Thierry Blu. PURE-LET image deconvolution. IEEE Transactions on Image Processing, 27(1):92-105, 2017. ISSN 1057-7149.

Ying Li, Jianglei Di, Wanqing Wu, Peng Shang, and Jianlin Zhao. Quantitative investigation on morphology and intracellular transport dynamics of migrating cells. Applied Optics, 58(34):G162-G168, 2019. ISSN 2155-3165.

Yuqian Li, Bruno Cornelis, Alexandra Dusa, Geert Vanmeerbeeck, Dries Vercruysse, Erik Sohn, Kamil Blaszkiewicz, Dimiter Prodanov, Peter Schelkens, and Liesbet Lagae. Accurate label-free 3-part leukocyte recognition with single cell lens-free imaging flow cytometry. Computers in Biology and Medicine, 96: 147-156, 2018. ISSN 0010-4825. 
J. Y. Liu, Y. Liu, P. Xiang, L. Pu, H. F. Xiong, C. S. Li, M. Zhang, J. B. Tan, Y. L. Xu, R. Song, M. H. Song, L. Wang, W. Zhang, B. Han, L. Yang, X. J. Wang, G. Q. Zhou, T. Zhang, B. Li, Y. B. Wang, Z. H. Chen, and X. B. Wang. Neutrophil-to-lymphocyte ratio predicts critical illness patients with 2019 coronavirus disease in the early stage. Journal of Translational Medicine, 18(1):1-12, 2020.

Laurens van der Maaten and Geoffrey Hinton. Visualizing data using t-SNE. Journal of Machine Learning Research, 9(Nov):2579-2605, 2008.

Stefano Managò, Carmen Valente, Peppino Mirabelli, Diego Circolo, Filomena Basile, Daniela Corda, and Anna Chiara De Luca. A reliable Raman-spectroscopy-based approach for diagnosis, classification and follow-up of B-cell acute lymphoblastic leukemia. Scientific Reports, 6:24821, 2016. ISSN 2045-2322.

Lisa Meintker, Jürgen Ringwald, Manfred Rauh, and Stefan W. Krause. Comparison of automated differential blood cell counts from Abbott Sapphire, Siemens Advia 120, Beckman Coulter DxH 800, and Sysmex XE2100 in normal and pathologic samples. American Journal of Clinical Pathology, 139(5):641-650, 2013.

Mustafa Mir, Zhuo Wang, Zhen Shen, Michael Bednarz, Rashid Bashir, Ido Golding, Supriya G Prasanth, and Gabriel Popescu. Optical measurement of cycle-dependent cell growth. Proceedings of the National Academy of Sciences, 108(32):13124-13129, 2011. ISSN 0027-8424.

Mustafa Mir, Basanta Bhaduri, Ru Wang, Ruoyu Zhu, and Gabriel Popescu. Quantitative phase imaging, volume 57, book section 3, pages 133-211. Elsevier, 2012. ISBN 0079-6638.

Vinod Nair and Geoffrey E Hinton. Rectified linear units improve restricted boltzmann machines. In Proc. 2010 International Conference on Machine Learning, page 432, 2010.

Mariam Nassar, Minh Doan, Andrew Filby, Olaf Wolkenhauer, Darin K. Fogg, Justyna Piasecka, Catherine A. Thornton, Anne E. Carpenter, Huw D. Summers, Paul Rees, and Holger Hennig. Label-Free Identification of White Blood Cells Using Machine Learning. Cytometry Part A, 95(8):836-842, 2019. ISSN 1552-4930.

Noga Nissim, Matan Dudaie, Itay Barnea, and Natan T. Shaked. Real-Time Stain-Free Classification of Cancer Cells and Blood Cells Using Interferometric Phase Microscopy and Machine Learning. Cytometry Part A, page 24227, 2020. ISSN 1552-4922.

Mengxuan Niu, Gang Luo, Xin Shu, Fuyang Qu, Shuang Zhou, Yiping Ho, Ni Zhao, and Renjie Zhou. Portable quantitative phase microscope for material metrology and biological imaging. Photonics Research, 8(7):1253-1259, 2020. ISSN 2327-9125.

Ashkan Ojaghi, Gabriel Carrazana, Christina Caruso, Asad Abbas, David R Myers, Wilbur A Lam, and Francisco E Robles. Label-free hematology analysis using deep-ultraviolet microscopy. Proceedings of the National Academy of Sciences, 117(26):14779-14789, 2020. ISSN 0027-8424.

Jadwiga Olsson, Anders Wikby, Boo Johansson, Sture Löfgren, Bengt-Olof Nilsson, and Frederick G Ferguson. Age-related change in peripheral blood T-lymphocyte subpopulations and cytomegalovirus infection in the very old: the Swedish longitudinal OCTO immune study. Mechanisms of Ageing and Development, 121(1-3):187-201, 2001. ISSN 0047-6374.

Yusuke Ozaki, Hidenao Yamada, Hirotoshi Kikuchi, Amane Hirotsu, Tomohiro Murakami, Tomohiro Matsumoto, Toshiki Kawabata, Yoshihiro Hiramatsu, Kinji Kamiya, Toyohiko Yamauchi, Kentaro Goto, Yukio Ueda, Shigetoshi Okazaki, Masatoshi Kitagawa, Hiroya Takeuchi, and Hiroyuki Konno. Label-free classification of cells based on supervised machine learning of subcellular structures. PLOS ONE, 14(1):e0211347, 2019.

Timothy O'Connor, Arun Anand, Biree Andemariam, and Bahram Javidi. Deep learning-based cell identification and disease diagnosis using spatio-temporal cellular dynamics in compact digital holographic microscopy. Biomedical Optics Express, 11(8):4491-4508, 2020. 
Rishikesh Pandey, Renjie Zhou, Rosalie Bordett, Ciera Hunter, Kristine Glunde, Ishan Barman, Tulio Valdez, and Christine Finck. Integration of diffraction phase microscopy and Raman imaging for label-free morphomolecular assessment of live cells. Journal of Biophotonics, 12(4):e201800291, 2019. ISSN 1864-063X.

YongKeun Park, Catherine A Best, Kamran Badizadegan, Ramachandra R Dasari, Michael S Feld, Tatiana Kuriabova, Mark L Henle, Alex J Levine, and Gabriel Popescu. Measurement of red blood cell mechanics during morphological changes. Proceedings of the National Academy of Sciences, 107(15):6731-6736, 2010. ISSN 0027-8424.

YongKeun Park, Christian Depeursinge, and Gabriel Popescu. Quantitative phase imaging in biomedicine. Nature Photonics, 12(10):578, 2018.

Gabriel Popescu. Quantitative phase imaging of cells and tissues. McGraw Hill Professional, 2011.

Gabriel Popescu, Takahiro Ikeda, Catherine Best, Kamran Badizadegan, Ramachandra R Dasari, and Michael S Feld. Erythrocyte structure and dynamics quantified by Hilbert phase microscopy. Journal of Biomedical Optics, 10(6):060503, 2005. ISSN 1083-3668.

Gabriel Popescu, Takahiro Ikeda, Ramachandra R. Dasari, and Michael S. Feld. Diffraction phase microscopy for quantifying cell structure and dynamics. Optics Letters, 31(6):775-777, 2006.

Gabriel Popescu, YoungKeun Park, Niyom Lue, Catherine Best-Popescu, Lauren Deflores, Ramachandra R Dasari, Michael S Feld, and Kamran Badizadegan. Optical imaging of cell mass and growth dynamics. American Journal of Physiology-Cell Physiology, 295(2):C538-C544, 2008. ISSN 0363-6143.

David Martin Ward Powers. Evaluation: from precision, recall and F-measure to ROC, informedness, markedness and correlation. Journal of Machine Learning Technologies, 2(1):37-63, 2011. ISSN 22293981.

Anuradha Ramoji, Ute Neugebauer, Thomas Bocklitz, Martin Foerster, Michael Kiehntopf, Michael Bauer, and Jurgen Popp. Toward a spectroscopic hemogram: Raman spectroscopic differentiation of the two most abundant leukocytes from peripheral blood. Analytical Chemistry, 84(12):5335-5342, 2012. ISSN 0003-2700.

Yair Rivenson, Tairan Liu, Zhensong Wei, Yibo Zhang, Kevin de Haan, and Aydogan Ozcan. PhaseStain: the digital staining of label-free quantitative phase microscopy images using deep learning. Light: Science E3 Applications, 8:23, 2019. ISSN 2047-7538.

Herbert Robbins and Sutton Monro. A stochastic approximation method. The Annals of Mathematical Statistics, 22(3):400-407, 1951. ISSN 0003-4851.

DongHun Ryu, Kyunghwan Kim, Hayeon Cho, Khoi Dao, Young Seo Kim, Daewoong Ahn, Hyun-Seok Min, Eui-Cheol Shin, and YongKeun Park. Label-free 3-D quantitative phase imaging cytometry with deep learning: identifying naive, memory, and senescent T cells. The Journal of Immunology, 204, 2020. ISSN 0022-1767.

Takaya Saito and Marc Rehmsmeier. The precision-recall plot is more informative than the ROC plot when evaluating binary classifiers on imbalanced datasets. PloS One, 10(3):e0118432, 2015. ISSN 1932-6203.

Xin Shu, Sameera Sansare, Di Jin, Kai-Yu Tong, Rishikesh Pandey, and Renjie Zhou. White Blood Cell Classification Using Quantitative Phase Microscopy Based Deep Learning. In Biophotonics Congress: Optics in the Life Sciences Congress 2019 (BODA,BRAIN,NTM,OMA,OMP), page DT3B.3. Optical Society of America, 2019.

Karen Simonyan and Andrew Zisserman. Very deep convolutional networks for large-scale image recognition. arXiv:1409.1556, 2015. 
L. Strbkova, P. Vesely, and R. Chmelik. Classification of Cells in Time-Lapse Quantitative Phase Image by Supervised Machine Learning. In Digital Holography and Three-Dimensional Imaging 2019, OSA Technical Digest, page Th3A.39. Optical Society of America, 2019.

Yuta Suzuki, Koya Kobayashi, Yoshifumi Wakisaka, Dinghuan Deng, Shunji Tanaka, Chun-Jung Huang, Cheng Lei, Chia-Wei Sun, Hanqin Liu, and Yasuhiro Fujiwaki. Label-free chemical imaging flow cytometry by high-speed multicolor stimulated Raman scattering. Proceedings of the National Academy of Sciences, 116(32):15842-15848, 2019. ISSN 0027-8424.

Christian Szegedy, Wei Liu, Yangqing Jia, Pierre Sermanet, Scott Reed, Dragomir Anguelov, Dumitru Erhan, Vincent Vanhoucke, and Andrew Rabinovich. Going deeper with convolutions. In Proc. 2015 IEEE Conference on Computer Vision and Pattern Recognition, pages 1-9, 2015.

Jecko Thachil and Imelda Bates. Approach to the Diagnosis and Classification of Blood Cell Disorders, book section 23, pages 497-510. Elsevier Health Sciences, 2017.

Matthias Ugele, Markus Weniger, Manfred Stanzel, Michael Bassler, Stefan W Krause, Oliver Friedrich, Oliver Hayden, and Lukas Richter. Label-free high-throughput leukemia detection by holographic microscopy. Advanced Science, 5(12):1800761, 2018. ISSN 2198-3844.

Hongda Wang, Yair Rivenson, Yiyin Jin, Zhensong Wei, Ronald Gao, Harun Günaydın, Laurent A Bentolila, Comert Kural, and Aydogan Ozcan. Deep learning enables cross-modality super-resolution in fluorescence microscopy. Nature Methods, 16(1):103-110, 2019. ISSN 1548-7105.

Cheng Ham Wu, Tzung Dau Wang, Chia Hung Hsieh, Shih Hung Huang, Jong Wei Lin, Szu Chun Hsu, Hau Tieng Wu, Yao Ming Wu, and Tzu Ming Liu. Imaging cytometry of human leukocytes with third harmonic generation microscopy. Scientific Reports, 6:37210, 2016. ISSN 2045-2322.

Jonghee Yoon, YoungJu Jo, Min-hyeok Kim, Kyoohyun Kim, SangYun Lee, Suk-Jo Kang, and YongKeun Park. Identification of non-activated lymphocytes using three-dimensional refractive index tomography and machine learning. Scientific Reports, 7(1):6654, 2017. ISSN 2045-2322.

Jingfang K Zhang, Yuchen R He, Nahil Sobh, and Gabriel Popescu. Label-free colorectal cancer screening using deep learning and spatial light interference microscopy (SLIM). APL Photonics, 5(4):040805, 2020. ISSN 2378-0967.

Guoan Zheng, Roarke Horstmeyer, and Changhuei Yang. Wide-field, high-resolution Fourier ptychographic microscopy. Nature Photonics, 7(9):739-745, 2013. ISSN 1749-4893. 